\title{
Eye color as an indicator of social rank in the fish Nile tilapia
}

G.L. Volpato, A.C. Luchiari,

C.R.A. Duarte, R.E. Barreto and G.C. Ramanzini
Research Center on Animal Welfare - RECAW Laboratório de Fisiologia e Comportamento Animal, Departamento de Fisiologia, Instituto de Biociências, Universidade Estadual Paulista, Botucatu, SP, Brasil
Correspondence

G.L. Volpato

Departamento de Fisiologia

IBB, UNESP

18618-000 Botucatu, SP

Brasil

Fax: +55-14-6821-3744

E-mail: volpato@ibb.unesp.br

Presented at the XVIII Annual Meeting of the Federação de Sociedades de Biologia Experimental, Curitiba, PR, Brazil, August 27-30, 2003.

Publication supported by FAPESP. ....................

Received April 25, 2003 Accepted September 5, 2003 .....................

\section{Abstract}

We investigated the association of eye color with the dominantsubordinate relationship in the fish Nile tilapia, Oreochromis niloticus. Eye color pattern was also examined in relation to the intensity of attacks. We paired 20 size-matched fish (intruder: $73.69 \pm 11.49 \mathrm{~g}$; resident: $75.42 \pm 8.83 \mathrm{~g}$ ) and evaluated eye color and fights. These fish were isolated in individual aquaria for 10 days and then their eye color was measured $5 \mathrm{~min}$ before pairing (basal values). Twenty minutes after pairing, eye color and fights were quantified for $10 \mathrm{~min}$. Clear establishment of social hierarchy was observed in 7 of 10 pairs of fish. Number of attacks ranged from 1 to 168 among pairs. The quartile was calculated for these data and the pairs were then divided into two classes: low-attack (1 to 111 attacks - 2 lower quartiles) or high-attack (112 to 168 attacks - 2 higher quartiles). Dominance decreased the eye-darkening patterns of the fish after pairing, while subordinance increased darkening compared to dominance. Subordinate fish in lowattack confrontations presented a darker eye compared to dominant fish and to the basal condition. We also observed a paler eye pattern in dominants that shared low-attack interactions after pairing compared to the subordinates and within the group. However, we found no differences in the darkening pattern between dominants and subordinates from the high-attack groups. We conclude that eye color is associated with social rank in this species. Moreover, the association between eye color and social rank in the low-attack pairs may function to reduce aggression.

The effects of social stress in fish have been well established. These effects change the internal state of the fish so that some processes are affected. For instance, social stress suppresses or decreases growth in subordinate fish (1), decreases resistance to diseases and abolishes reproduction (2). Moreover, high variability in physiological parameters of grouped territorial fish has also
Key words

- Aggression

- Eye color

- Social hierarchy

- Social stress

- Fish

- Oreochromis niloticus ...................... been attributed to the different levels and types of social interaction experienced by each fish (3). Therefore, identification of fish social status in a group is an important goal for further studies and management of these animals.

Social status in territorial fish is usually identified by analysis of the agonistic profile: the dominant fish attacks while the sub- 
ordinate fish flees (4). As a consequence, subordinates and dominants are physiologically and behaviorally affected, with the occurrence of changes that may be useful for inferences about their social rank (5).

Thus, changes in color have been recognized as a reliable indicator of social status in some species. Subordinate status is usually associated with a darker appearance while the dominants are paler (6). This association between social rank and body color is also assumed to correspond to intraspecific signals that may decrease aggression and establish hierarchy (7). Body color is the characteristic most extensively studied $(7,8)$, but recent investigations have concentrated on eye color $(9,10)$. Eye color is certainly easy to identify and its adequacy as an indicator of social rank in other fish species should be considered.

In the present study we addressed this question, testing whether eye color is a reliable indicator of social status in the Cichlidae fish Nile tilapia, Oreochromis niloticus (L.). This is one of the most important species in tropical aquaculture and an important fish model for studies on social stress $(1,3,11)$.

This study agrees with ethical principles in animal research adopted by the Brazilian College of Animal Experimentation and was approved by the Biosciences Institute (UNESP) Ethics Committee for Animal Research (protocol No. 006/03).

Juveniles of the fish Nile tilapia (Oreochromis niloticus) housed for about one year in indoor 1200-liter tanks (about 2 fish/liter) were used as the stock population. During this period, temperature averaged $25 \pm 1^{\circ} \mathrm{C}$, and water was maintained saturated with oxygen, with low levels of ammonia $(<0.5$ $\mathrm{ppm})$ and nitrite $(<0.05 \mathrm{ppm})$. The photoperiod was from 6:00 to $18: 00 \mathrm{~h}$. Food was offered once a day in excess (more than 5\% of the fish biomass).

The study consisted of pairing the fish and quantifying eye color and confrontations in each pair. Twenty Nile tilapia speci- mens were isolated at random (1 fish/aquarium) in glass aquaria $(28.0 \times 11.4 \times 19.6 \mathrm{~cm})$ for ten consecutive days and food was provided once a day in the morning. On the 11th day, the eye color pattern of each fish was measured five consecutive times at 1-min intervals, with the mean taken to be the basal value. Immediately after these measurements, fish of similar size were paired; an intruder fish $(73.69 \pm 11.49 \mathrm{~g}, 13.6 \pm 0.70 \mathrm{~cm})$ was introduced into the aquarium of a conspecific $(75.42 \pm 8.83 \mathrm{~g} ; 14.40 \pm 0.65 \mathrm{~cm})$, imposing a resident-intruder situation to assure a clear dominant-subordinate relationship. To control handling effects, the resident fish was also taken out and reintroduced into the aquarium at the time of introducing the intruder fish. After 20-min pairing, the total number of agonistic attacks was recorded continuously for $10 \mathrm{~min}$ and eye color was recorded at 1-min intervals. The fish that attacked and did not leave the confrontation area and that also chased the opponent were considered to be dominant. The effect of hierarchical rank on eye color was determined by changes in eye color pattern in 5-min blocks (basal values and 20 to 24 and 25 to $29 \mathrm{~min}$ after pairing). Fish were identified individually by cutting their caudal fin in different manners. Mean water temperature was $24.75 \pm 1.06^{\circ} \mathrm{C}$ and oxygen concentration ranged from 7.26 to $7.49 \mathrm{mg} /$ 1. The photoperiod was from 6:00 to $18: 00 \mathrm{~h}$ and the observations were carried out from 8:00 to $14: 00 \mathrm{~h}$.

The Nile tilapia eye has a central black pupil surrounded by the iris and sclera, both clearly ranging from pale to dark. Pilot observations showed that color change in this area is gradual and not marked by stripes. Moreover, eye color is independent of the body stripes since darkened stripes can appear without the occurrence of a darkened eye and vice-versa. Eye darkening has been studied in other fish using gradual color patterns transformed into scores (10). Here, eye color was quantified as percent dark- 
ened area of both the iris and sclera, thus reflecting darkening. The circular area of the eye was drawn and 4 diameters were used to divide the eye into 8 parts of equal areas. Observations consisted of filling in each area representing the darkened pattern of the eye (dark or white, thus calculating the percentage of black area). Although only one observer recorded eye color, a double-blind scheme was adopted: one examiner observed the eye color and another the social interactions of the fish, and these data were subsequently matched.

Clear establishment of dominance hierarchy was observed in 7 of 10 pairs, which were then the subject of the subsequent analyses. Subordinate fish stayed close to the surface of the aquarium. Even though confrontations were not recorded individually for each fish, but only for the pair, fights were mostly initiated by the dominant fish. Moreover, in 6 of 7 pairs the resident fish was the dominant one.

The wide range of fights (from 1 to 168) detected among the 7 groups where fights occurred was used to assess the relationship between fights and hierarchical rank effect on eye color. No significant correlation occurred between number of fights and percent darkening (Spearman test: subordinates, $\mathrm{P}=$ 0.28 ; dominants, $\mathrm{P}=0.80$ ). However, a clear effect was observed (Figure 1) when the pairs were subdivided into two classes by analysis of the quartiles, in which the first and second quartiles were the lower-attack pairs (1 to 111 attacks) and the third and fourth quartiles the higher-attack pairs (112 to 168 attacks). These data are illustrated in Figure 1 and show that the hierarchical effect on eye color was restricted to the lowattack pairs (compare top and middle panel).

Figure 1 (top panel) shows that the basal values of eye darkening were statistically similar between the hierarchical ranks, but after pairing the eyes of the subordinate fish darkened and the eyes of the dominants paled (Friedman's test between basal and pairing
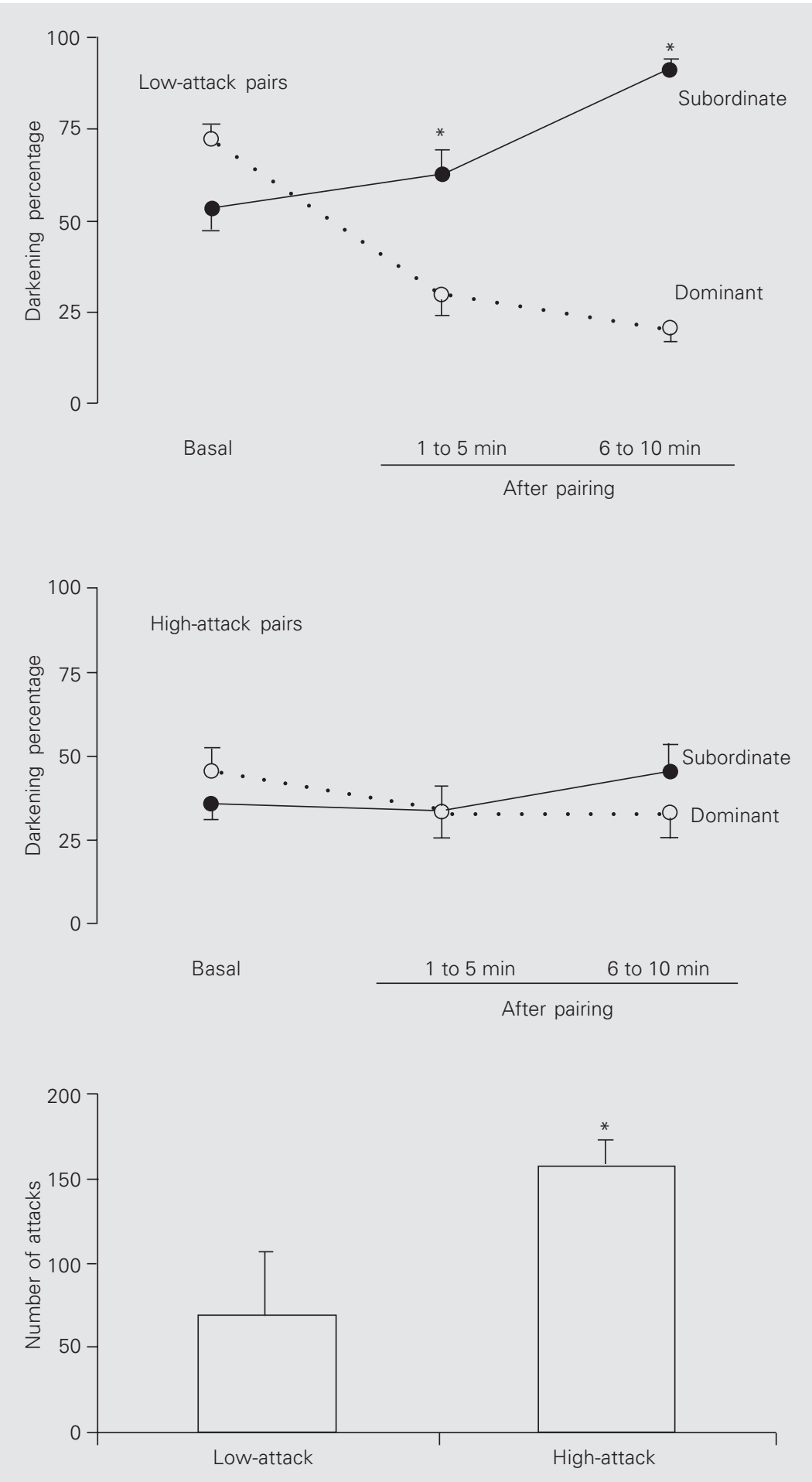

Figure 1. Effect of hierarchical rank on eye-darkening pattern in the high-attack and lowattack group of Nile tilapia. Data are reported as mean percent darkness $\pm S D$, which indicates the black area (\%) of sclera and iris region. Low-attack group, $\mathrm{N}=4$, and highattack group, $N=3$. ${ }^{*}$ Difference over time $(P=0.0001$, Friedman test) and between ranks after pairing $(\mathrm{P}<0.05$, Mann-Whitney U-test $)$. 
values: $\mathrm{P}=0.0001$ for dominants; $\mathrm{P}=0.044$ for subordinates). The eyes of subordinates were darker compared with the dominants at the same time (Mann-Whitney U-test: $\mathrm{P}<$ 0.05). Moreover, only the subordinate fish showed increased eye darkening during the time of pairing.

The data reported above corroborate the fact that, in the Nile tilapia, eye color is affected by social status. The subordinate fish exhibit darker eyes and the dominants paler eyes. This association has also been described for salmonids $(9,10)$. This observation also supports Falter's (8) qualitative observation that in the Nile tilapia the subordinates show darker body color than the dominants. At least in salmonids, eye color has been associated with body color (7).

The strong difference between the eye color of dominants and subordinates after 10-min pairing indicates that this parameter is a reliable acute indicator of social status in this species. The subordinate's eye has more than $80 \%$ of its area darkened, while this color in the dominant fish is less than $25 \%$. Although our results are restricted to paired fish, ad hoc observations in groups larger than two fish reveal fish with eye color ranging from dark to pale. This should contribute to an easy identification of these fish in a group, thus facilitating selection of individuals for different purposes.

Another interesting aspect revealed by this study is that the socially mediated change in eye color is restricted to the pairs in which a low number of attacks occurred (Figure 1). Despite the small number of pairs considered, the very strong effect detected and the statistical significance obtained validate this association. However, the darkening of the subordinate's sclera has been associated with higher frequencies of received attacks in salmonids (10). In the present study, however, agonistic events were quantified only 10 min after paring so that the eye color may have been an effect of stronger fights during this pre-observation period. The low-attack pairs are those in which hierarchy was clearly established previously and thus the socially mediated eye color pattern was evident.

Fish in the high-attack pairs, on the other hand, could have been fighting up to the end of the observations, with a less clear dominant-subordinate relationship. However, even in these high-attack pairs, social hierarchy could be identified, thus suggesting that eye color may be linked to other social aspects.

Eye and body color variations in fish have been interpreted as dominant-subordinate signals that affect fights. Accordingly, color changes signal to the opponent the rank of the fish, thus decreasing aggressive agonistic interactions (6). This same interpretation can be extended to the results of the present study. In some pairs the eye color signal latency was shorter, thus decreasing fights (low-attack pairs). In contrast, in the high-attack pairs the absence of this clear signal may have contributed to the persisting agonistic confrontation. The high level of aggression in the high-attack pairs and the absence of eye color differentiation may suggest that, under these circumstances, subordinates are somehow different from those of the low-attack pairs, suggesting that hierarchies based on a fight-flight paradigm may not represent the complete picture.

\section{Acknowledgments}

The authors thank Mr. Danilo Pinhal for helpful technical assistance. 


\section{References}

1. Volpato GL \& Fernandes MO (1994). Social control of growth in fish. Brazilian Journal of Medical and Biological Research, 27: 797-810.

2. Moberg GP \& Mench JA (2000). The Biology of Animal Stress: Basic Principles and Implications for Animal Welfare. CAB International, New York.

3. Alvarenga CDM \& Volpato GL (1995). Agonistic profile and metabolism in alevins of the Nile tilapia. Physiology and Behavior, 57: 7580.

4. Dawkins R (1976). Hierarchical organization: a candidate principle for ethology. In: Bateson PPG \& Hinde RA (Editors), Growing Points in Ethology. Cambridge University Press, London, England.

5. Haller J (1995). Biochemical background for an analysis of costbenefit interrelations in aggression. Neuroscience and Biobehavioral Reviews, 19: 599-604.

6. Beeching SC (1995). Colour pattern and inhibition of aggression in the cichlid fish Astronotus ocellatus. Journal of Fish Biology, 47: 50-58.
7. O'Connor KI, Metcalfe NB \& Taylor AC (1999). Does darkening signal submission in territorial contests between juvenile Atlantic salmon, Salmo salar? Animal Behaviour, 58: 1269-1276.

8. Falter U (1987). Description des patrons de coloration chez Oreochromin niloticus (L.) (Teleostei: Cichlidae). Annales de la Société Royale de Zoologie Belge, 117: 201-219.

9. O'Connor KI, Metcalfe BN \& Taylor AC (2000). Familiarity influences body darkening in territorial disputes between juvenile salmon. Animal Behaviour, 59: 1095-1101.

10. Suter HC \& Huntingford FA (2002). Eye color in juvenile Atlantic salmon: effects of social status, aggression and foraging success. Journal of Fish Biology, 61: 606-614.

11. Barcellos LJG, Nicolaiewsky S, Souza SMG \& Lulhier F (1999). The effects of stocking density and social interaction on acute stress response in Nile tilapia Oreochromis niloticus (L.) fingerlings. Aquaculture Research, 30: 887-892. 